\title{
La idealización de la profesión de maestro y los límites de la formación docente: análisis de un caso
}

Joaquín Paredes-Labra

Universidad Autónoma de Madrid. España. joaquin.paredes@uam.es

Alicia Kachinovsky Melgar

Universidad de la República. Uruguay.

alicia.kachinovsky@gmail.com

Recibido: 4/3/2020

Aceptado: 13/7/2020

Publicado: 29/1/2021

\section{Resumen}

Este trabajo aborda la presencia de idealización o de formas estereotipadas de imaginar el ejercicio de la profesión de educador en el marco de su formación inicial y las consecuencias que dicha consideración tiene para el desarrollo de la docencia en la formación de profesores y en el futuro de los actuales estudiantes de Maestro. Se desarrolla un estudio de caso. El contexto es un proceso de acompañamiento para la mejora de la propia docencia. Se utiliza una metodología mixta, cuantitativa y cualitativa. Se analizan las percepciones sobre la figura del docente de dos grupos de clase de Maestro de Educación Primaria con los que trabaja un profesor. Se realiza una encuesta a los estudiantes. Se dialoga sobre los resultados con el docente responsable y el grupo. La triangulación de la información mediante diferentes técnicas permite la génesis de un informe balanceado sobre la idealización. Los resultados denotan una adherencia de los futuros maestros a un deber ser profesional de elevados fines, una perspectiva muy centrada en la enseñanza para la transmisión, que ignora tanto las características del aprendizaje como cuestiones didácticas que podrían mejorarla y que dificultan la preparación como docente.

Palabras clave: características del maestro; educación primaria; creencias; motivaciones; formación del profesorado

Resum. La idealització de la professió de mestre i els límits de la formació docent: anàlisi d'un cas

Aquest treball aborda la presència d'idealització o de maneres estereotipades d'imaginar l'exercici de la professió d'educador en el marc de la seva formació inicial i les conseqüències que aquesta consideració té en referència al desenvolupament de la docència en la formació de professors i en el futur dels actuals estudiants de Mestre. Desenvolupa un estudi de cas. El context és un procés d'acompanyament per millorar la pròpia docència. Utilitza una metodologia mixta, quantitativa i qualitativa. Analitza les percepcions sobre la figura del docent de dos grups de classe de Mestre d'Educació Primària amb qui treballa un professor. Es realitza una enquesta als estudiants. Es dialoga sobre els resultats amb el professor responsable i el grup. La triangulació de la informació amb diferents 
tècniques permet generar un informe de balanceig sobre la idealització. Els resultats denoten una adherència dels futurs mestres al fet d'haver de ser professionals d'elevats fins, amb una perspectiva molt centrada en l'ensenyament per a la transmissió que ignora tant les característiques de l'aprenentatge com qüestions didàctiques que podrien millorar-la i que dificulten la preparació com a docent.

Paraules clau: característiques del mestre; educació primària; creences; motivacions; formació del professorat

Abstract. The idealization of the teaching profession and the limits of teacher training: A case analysis

This work addresses the presence of idealization or stereotyped ways of imagining the exercising of the profession of teacher, within the framework of initial teacher training, and the consequences it has for the development of teaching in teacher training and for the future of today's trainee teachers. A case study is developed. The context is a support process for teaching improvement. A mixed, quantitative and qualitative methodology is used. The perceptions of the teacher figure in two primary school teacher class groups with which a teacher works are analyzed. A student survey is conducted. The results were discussed with the teacher in charge and the group. The triangulation of information with different techniques allowed the genesis of a balanced report on idealization. The results denote the adherence of future teachers to a duty to be a professional with high aims, a perspective highly focused on teaching for transmission, which ignores both the characteristics of learning and teaching issues that could improve it, and that hinder teacher training.

Keywords: teacher characteristics; primary education; beliefs; motivations; teacher training

\section{Sumario}

$\begin{aligned} \text { 1. Introducción } & \text { 4. Conclusiones } \\ \text { 2. Metodología } & \text { Referencias bibliográficas } \\ \text { 3. Resultados } & \end{aligned}$

\section{Introducción}

Los trabajos sobre formación inicial de docentes preocupan de forma paralela a los procesos de reforma educativa, pues dicha formación es uno de los factores de garantía de tales procesos.

Durante las décadas de 1980 y 1990 se realizaron numerosos trabajos para comprender los problemas y las necesidades de la formación inicial (Joyce y Clift, 1984; Benejam, 1986; Lundgren, 1988; Santos Guerra, 1993), si bien en la última década se han puesto de manifiesto dificultades añadidas (Medina y Pérez-Cabrera, 2017), derivadas de dilemas epistemológicos entre los propios formadores sobre el tipo de conocimiento que nutre la formación.

Este viejo debate sobre las relaciones entre la teoría y la práctica no deja de manifestar el malestar por la permanente reforma, el cuestionamiento a los 
profesores en ejercicio y la necesidad de recuperar el conocimiento producido en las prácticas de enseñanza.

También se pone en evidencia el problema de que la profesión de maestro parece algo que es cuestión de tiempo llegar a dominar, puesto que es el producto del ensayo y el error (Esteve, 1998), y de que la formación académica para llegar a ser docente es un trámite, algo sin importancia o innecesario, pues, según el parecer de algunos profesores en ejercicio, se aprende simplemente practicando la enseñanza.

Uno de los temas que preocupan es quiénes son los seleccionados para ser formados y sus imaginarios. No en vano, Schön (1983) propone que para aprender a enseñar hay que exorcizar aprendizajes previos sobre lo que significa estar escolarizado. Hay que acabar con los estereotipos sobre la docencia. Los futuros maestros han pasado escolarizados entre 12 y 18 años (en la educación infantil, primaria y secundaria). Su vivencia condiciona el «deber ser» o el "podría ser» de las aulas, ven la docencia a través de los ojos de su propia experiencia vital. Identificar sus fantasmas a través de la escritura de biografías y diarios es la base para poder empezar a aprender y reflexionar sobre múltiples condicionantes de la práctica.

Como señalan algunos estudios (Sánchez Lisson, 2009; Gallardo y Vázquez, 2016; San Román, 2019), hay una plétora de motivaciones y condicionantes sociológicos, económicos e históricos para llegar a elegir esta formación. El espacio profesional de los maestros es un campo deseable para numerosos estudiantes españoles. El origen subalterno, rural y femenino de quienes mayoritariamente se formaron durante mucho tiempo como enseñantes en España da cuenta de un imaginario de mejora de posición social a través de la docencia.

Una forma estereotipada de pensar la formación docente adopta visos de idealización (Laplanche y Pontalis, 1981). Se atribuye al objeto una perfección y una valoración de la que carece o una inflación de sus cualidades reales. Es preciso considerar cómo se vería afectado el desempeño profesional de los futuros maestros, pues la realidad escolar que pone en jaque con insistencia los ideales e impone límites a la práctica docente puede suponer, en el ejercicio del trabajo, que las aspiraciones iniciales sean reemplazadas por un sentimiento de derrota y futilidad.

Muchos aprendices de maestro anteriores a los actuales han podido simultanear una actividad laboral junto con sus estudios. Así, una investigación ha explorado el compromiso de los alumnos con la carrera a partir de la sospecha de que es mayor (al menos motivacionalmente) el de quienes tienen más obligaciones laborales simultáneas a su formación como docentes (Garrote y Jiménez-Fernández, 2018). El número de estudiantes con compromisos laborales puede alcanzar dos tercios del colectivo en formación. De los dos perfiles de futuros maestros con compromiso laboral paralelo se observa que los que tienen jornada parcial están más motivados y muestran un sentido de autoeficacia mayor en la formación inicial como maestros, en tanto que los que tienen empleos de cuarenta horas semanales muestran más sensibilidad en 
referencia a las dificultades que plantea el estudio, y son proclives al fracaso y al abandono. Estas vivencias formativas tienen que condicionar de algún modo la caracterización de la profesión.

Otra dimensión motivacional está en el maestro venerado o, en negativo, el maestro que no se quiere ser. Al respecto, el estudio de Esteban y Mellen (2016) identifica tres ideales entre los docentes en formación: el personal y auténtico, el que transmite historias y el preocupado por la motivación de sus estudiantes. El estudio revela que los estudiantes se pronuncian mayoritariamente por un ideal de maestro personal y auténtico, el que resulta encumbrado en las biografías de grandes personajes y el que se ensalza en películas exitosas. Ahora bien, lo preocupante, siguen los autores, es la escasa importancia que se concede a la mirada sobre sí mismo, particularmente entre mujeres, su crecimiento, desarrollo personal y autoconocimiento. Es una falta de perspectiva profesional suplantada por el dominio de unas supuestas técnicas y por el mero entusiasmo, lo que preocupa a los investigadores.

Por otra parte, la investigación señala, al comparar respuestas de estudiantes del primer y del último año de formación, que los ideales se atenúan con el paso del tiempo. Así, la formación inicial deja una huella y obliga a cuestionar determinadas creencias.

La formación inicial prepara para el proceso de profesionalización, si bien pone en marcha un enfrentamiento entre un plano en cierto modo ideal y otro más funcional, pues casi inmediatamente después de verse más reflexivos los estudiantes manifiestan que desconocen la vida del centro educativo en su formación inicial (Paredes, Esteban y Fernández-Prieto, 2016), lo que equivale a negar su capacidad para comprender la profesión para la que han sido formados. Parecen necesitar trucos o recetas.

En síntesis, formar un maestro abierto al cambio (Medina y Pérez-Cabrera, 2017) se convierte en un problema. Cabe preguntarse si los futuros docentes esperan una formación más funcional y que obedezca a un ideal de enseñante, y si se está recibiendo otra más crítica y por lo tanto más inquietante.

\section{Metodología}

El estudio que se presenta se interesó por conocer el grado de ajuste entre el conjunto de motivaciones de aprendizaje de los alumnos de una materia - TIC para la Sociedad Digital — en la titulación de Maestro de Educación Primaria de una universidad española, así como el tipo de prácticas promovidas en la misma.

Este estudio forma parte de otro más amplio creado para la mejora de la docencia en la materia, que parte de las preocupaciones expresadas por el profesor de la asignatura sobre los resultados de las encuestas de satisfacción de sus estudiantes en pasados cursos y ciertas limitaciones de los resultados académicos.

Se organizó un proceso de acompañamiento de sus clases (Sancho, Hernandéz, Larraín y Montané, 2011), con la idea de analizarlas y optimizarlas. Estos procesos de acompañamiento pueden formar parte de la fase diagnósti- 
ca de procesos de mejora de la enseñanza en la forma de investigación-acción (Latorre, 2003), de desarrollo profesional (Tardif, 2004) o de análisis de trayectorias profesionales (Goodson, Biesta, Tedder y Adair, 2010), o bien ser, simplemente, una investigación respetuosa con los saberes de quien los comparte (Hernández, 2008).

Se tuvo que hacer en el tiempo en el que una amiga crítica se pudo liberar de sus propias clases en la Universidad de la República, en Uruguay, entre el tercer y el cuarto mes del segundo semestre de España (mayo), durante el curso 2018-2019, cuando estaba finalizando. El momento de realización del estudio no deja de ser una fotografía de lo que ocurre en la clase cuando acaba un semestre, lo que posiblemente supone una limitación en las aportaciones del caso.

En el acompañamiento se incluía la recogida de información mediante diversas técnicas. Se realizaron entrevistas con el profesor, diálogos en el que emergía su proyecto docente, y se tuvieron en cuenta los resultados de las encuestas de satisfacción del grupo y su propio diario, que es público, pues se sustantiva en un blog en el que reflexiona sobre su práctica. Asimismo, se desarrolló una observación no participante y asistemática basada en la presencia en el aula durante un mes, en las clases que se desarrollan durante dos días de la semana. Todos estos datos permitieron contextualizar el trabajo.

Para completar la recogida de información se pasó una encuesta entre los estudiantes. Con este propósito se realizó un cuestionario ad hoc basado en otros similares elaborados por el profesor en pasados cursos, a fin de conocer a sus alumnos (género, edad, lugar de residencia, estudios previos, nivel educativo de los padres, idiomas y actividad laboral simultánea a la carrera) y sus motivaciones (razón por haber elegido ser maestro, materias preferidas). Se les añadieron preguntas sobre su percepción de la docencia, provenientes de un material utilizado en otro ámbito de formación docente (Universidad de la República) por la profesora invitada, y algunas preguntas abiertas. En concreto, se procuró detectar qué temáticas o asignaturas les habían resultado más interesantes, qué lugar ocupaba la tecnología educativa entre ellas, qué cualidades apreciaban en un docente en general y en el profesor universitario en particular, cuáles eran los cinco atributos que valoraban en un estudiante de magisterio y cómo se imaginaban en el ejercicio de dicha profesión.

Los participantes fueron los alumnos de la asignatura TIC para la Sociedad Digital, en los dos grupos de docencia en que imparte clase el profesor acompañado.

Los estudiantes del grupo 102 de primero de Maestro de Educación Primaria estaba constituido por 15 varones y 17 mujeres. La mayoría tenían entre 18 y 20 años. Muchos vivían con sus padres, aunque uno de cada cinco disponía de otra vivienda (vivía solo, con amigos o en una residencia). Algo menos de la mitad procedía de otros estudios. Ocho de cada diez eligieron la carrera en primera opción. Provenían a partes iguales de los cinco territorios de la región (la capital; una ciudad colindante con la universidad, y comarcas al sur, al este y al oeste de la ciudad). Dos de cada tres trabajaban desde unas horas hasta una jornada completa. 
Los estudiantes del grupo 112 de primero de Maestro de Educación Primaria estaba constituido por 33 estudiantes, 9 varones y 21 mujeres (hay tres que prefieren no identificarse por género al responder al cuestionario). La mayoría, 25, tenían entre 18 y 20. Mayoritariamente vivían con sus padres (salvo dos), nueve de cada diez hizo bachillerato para el acceso, tres de cada cuatro eligieron la carrera en primera opción. Dos tercios vivían en las proximidades y un tercio procedía de los lugares más alejados de la universidad. Solo trabajaba uno de cada tres.

Como nota adicional conviene comentar que existieron diferencias en la forma en que cada uno de los grupos contestó el cuestionario y se involucró en él. Mientras que en el 102 ningún estudiante escribió nada en el último apartado ("Comentarios y sugerencias que quiera compartir»), fueron seis los que sí lo hicieron en el grupo 112. Una estudiante llegó a afirmar: «Este cuestionario me ha hecho ver cómo tengo que ser para llegar a ser buena docente».

Se realizó un análisis descriptivo de las encuestas y una comparación de los resultados entre ambos grupos. La información recogida en las observaciones también fue categorizada y organizada en un relato, con una comparación entre los dos grupos participantes y el resto de las fuentes.

La redacción y el debate de informes preliminares con el profesor fue fundamental para clarificar y comprender estas prácticas en un marco más amplio que permitiera la mejora de su docencia.

Asimismo, los resultados de la encuesta fueron debatidos con cada grupo con la idea de generar una devolución formativa complementaria sobre la propia práctica, que les hiciera conscientes de la formación recibida y del proceso en el que se había embarcado su profesor.

En este trabajo se hace referencia a uno de los rasgos que emergieron, la idealización de la profesión de maestro y las consecuencias que ello comporta para el desarrollo de la docencia en la formación de profesores.

\section{Resultados}

\subsection{Perspectiva sobre el docente: posicionamiento subjetivo y vinculo entre el maestro y el aprendiz}

La proyección de la representación de sí mismos como docentes que más insiste discursivamente en estos estudiantes, tanto en varones como en mujeres, es la de ellos como agentes de cambio (particularmente en el grupo 102, casualmente el menos proclive a caracterizar el resto de los aspectos de su práctica), ideal que formulan de distintas maneras, entre las que destacan modificar la escuela o la educación tradicional y actual e innovar. Algunos lo expresan muy directamente, y otros, a través de planteos que permiten inferir tal circunstancia. Varios ponen el acento en el carácter placentero que debería tener la clase y aspiran a crear espacios disfrutables. La pretensión de aportar a la creación de una escuela deseable está muy presente en un alto número de respuestas. 
El profesor de la materia sostiene en las entrevistas, sin embargo, que no observa una particular implicación en trabajar para con la comunidad (una de las características de las tecnologías para conectar la escuela y la comunidad), incluso cuando está siendo objeto de los proyectos que han estado desarrollando los estudiantes. Los proyectos con tecnología son percibidos, manifiesta, como meras tareas. Entonces, el profesor se pregunta cómo se va a producir el cambio sin cuestionar la forma de relacionarse con el conocimiento y la comunidad.

Con esta misma perspectiva imaginan una figura docente que dispone de ciertas actitudes o cualidades, a saber: escuchar al otro, ser tolerante, empático, comprensivo, cultivar un aula inclusiva atendiendo a la singularidad de cada integrante y reconocer sus fortalezas. El ejercicio de la profesión a desempeñar comporta ilusión, amor y empeño, lo que supone un compromiso afectivo no menor. Desde este punto de vista, no es extraño que concite perplejidad o inquietud en quien lo define como una «enorme responsabilidad». En efecto, se concibe un docente comprometido con su tarea, pero también muy agobiado por sus responsabilidades.

Esta misma reacción emotiva desencadena distintas estrategias defensivas. Ello se manifiesta a través de la verbalización de meros interrogantes que no se acompañan de respuestas tentativas sobre sí mismos como futuros maestros o sobre los niños que tendrán a su cargo. En casos similares tampoco logran crear una hipótesis sobre la "clase perfecta» que querrían construir. Representar al maestro apelando al sentido del humor («maestro humorístico») resulta una estrategia más efectiva.

Ellos mismos manifiestan elevadas exigencias respecto al oficio elegido: un maestro capaz de "conseguir la motivación necesaria», de "llegar a los niños", de brindar «ayuda». Las declaraciones de estos futuros docentes no reparan en los contenidos a enseñar ni se detienen en los métodos a usar. Aunque en cierto momento se utiliza en forma indistinta el concepto de enseñar y el de formar, apuestan por una educación humanista, es decir, están preocupados por forjar personas y sembrar valores (entre ellos se subraya el de tener una postura inclusiva).

El aspecto asistencial se hace presente cuando dos varones califican al docente de guía o cuando dos varones y cuatro mujeres lo definen como alguien que ayuda a sus alumnos. Emerge así la fantasía de que hay personas necesitadas o carentes de atenciones a quienes hay que atender.

Hasta acá se ha resumido y procesado todo lo que ha sido considerado por el grupo como aquello que, en su condición de maestros, estarían en condiciones de brindar. No obstante, en algunos estudiantes emerge lo que podrían recibir. Se trata, posiblemente, de una suerte de retribución anhelada. Y ello es expuesto de distintos modos: llegar a ser el «mejor profesor del mundo", ser admirados y recordados, constituir un modelo a emular u obtener una suerte de realización laboral («mi futuro») y proyectarse hacia un mundo adulto. La necesidad de dejar una marca o huella en las demás personas parece remitir a la expectativa de prolongarse en el otro. 
El quehacer docente queda asociado en algunos estudiantes a la felicidad y a la alegría: «un futuro laboral que me hará feliz». Estos sentimientos, vinculados a «lo que me gusta», dan testimonio de la puesta en juego de un deseo. Debe tomarse en cuenta que tal deseo es dirigido a un objeto enaltecido, ajeno a su confrontación con la realidad en la que deberá insertarse y operar, pero también ajeno al síndrome del trabajador quemado, en la línea del estudio de Esteban y Mellen (2016). Predomina, por tanto, una fuerte idealización de la función docente.

En el discurso de estos jóvenes impera el deber ser, como puede apreciarse en expresiones que emplazan el esfuerzo, que reclaman una enseñanza «adecuada» que se atreven a "garantizarles un buen ambiente en el centro durante el curso". Lejos del gesto espontáneo descrito por Winnicott (1992), concomitante a un verdadero self en acción, se cae en el riesgo de que ese deber ser los atrape y no les permita responder creativamente a las dificultades del aula.

No es de extrañar que dichas idealizaciones se vinculen a añoranzas o a frustraciones infantiles, como puede apreciarse en el siguiente ejemplo de un estudiante del grupo 112 («ser el profesor que siempre quise tener cuando era pequeña»), lo cual reforzaría la propuesta de pensar la docencia como un modo de reparar en el otro las carencias sufridas por uno mismo. Solo un varón se suma a estas elevadas expectativas, al imaginar «un colegio perfecto». Podría plantearse entonces que, a despecho de lo afirmado anteriormente, el anhelo de producir cambios sustantivos está implícito en las idealizaciones reveladas. El cambio parece referirse a su crecimiento como profesores ideales. En su visión como docentes está ausente la innovación que podría ocurrir con tecnología.

\subsection{Perspectiva sobre los niños}

Junto a la idea de cambiar la educación, como perspectiva profesional, se reitera la aspiración de un futuro en que los niños no irían «obligados» a la escuela. El objetivo abrazado es manifestado a veces en forma explícita y otras veces se puede inferir. En un formato extremo es proclamado como el compromiso de alcanzar «una educación soñada por todas las niñas y los niños». Más factible resulta el designio de promover un clima de trabajo en que los alumnos «se respeten entre ellos».

En el grupo 112 la población infantil es aludida fundamentalmente como punto de partida del vínculo con el maestro. A modo de ejemplo, un estudiante imagina el siguiente escenario: «un grupo de niños y niñas con los que me llevo bien». Se vislumbra un docente "cercano», que brinda "cariño" y «atención», es empático y paciente.

Los estudiantes con los que se ha trabajado no parecen jerarquizar el contexto doméstico de los niños con los que habrán de desarrollar su labor docente. En el grupo 102 las familias únicamente se mencionan en una única oportunidad. Sin embargo, solo se espera recibir su «aprecio», es decir, no se las visualiza condicionando en alguna medida la educación de sus hijos. 
En el grupo 112 el deseo de ser maestro es conferido a estar con los niños. Ningún varón se ocupa del tema. En cuanto a las estudiantes mujeres, si bien el deseo de enseñar es enunciado en una ocasión («me imagino con muchas ganas»), en los otros casos se hará referencia al deseo de saber de los escolares, y ese deseo dependerá de ellos mismos, de su capacidad de manifestarlo: les gusta aprender porque están «contentos» y "porque se lo pasan bien/disfrutan» o porque enseñarán como les "habría gustado» que les enseñasen. Las dos personas que no se definen como varones o mujeres coinciden en preconizar un clima de «diversión» que acompañe al trabajo.

La impresión del profesor al dialogar sobre la perspectiva metodológica en la que están participando los estudiantes es que conciben la actividad con niños como mera transmisión de información. Las metodologías transmisivas parecen ser el único modelo metodológico. Las tecnologías se convierten entonces en instrumentos para la transmisión. Se silencian otras metodologías, como el juego y la cooperación, que se podrían implementar con tecnologías. De todas las fases en que puede dividirse la acción educativa de los docentes (preparación de la clase, preparación motivacional para la clase, presentación de información, presentación de tareas, desarrollo de trabajo individual o en grupos, evaluación) quizá es la preparación motivacional la única que se percibe como fundamental. El aspecto motivacional es, efectivamente, el tótem de los estudiantes.

\subsection{Perspectiva sobre el aprendizaje}

En las respuestas recogidas se detecta una ligera alusión al aprendizaje de los niños. En alguna de ellas se lo imaginan lúdico y divertido, incluso ostentando la cualidad de disfrutable. En consonancia con este contexto se evoca un aula solidaria, colaborativa y el trabajo en grupo. Corolario de lo anterior se llega a decir que «todos aprenden de todos, incluido el profe».

Los varones ni siquiera nombran al aprendizaje. En la sección de los comentarios, sin embargo, uno afirma que ellos cursan «una carrera preciosa, con un mundo entero por aprender y por mejorar». Una de las dos personas que prefieren no definir si son varones o mujeres defiende la idea de que los niños aprendan en un marco de diversión. No es muy distinto lo que plantean dos de tres estudiantes de sexo femenino. Hablan de un «buen aprendizaje» o supeditan que a sus alumnos les guste aprender a pasarlo bien («porque se lo pasan bien»). La tercera joven apuesta por un aprendizaje personalizado: «Me imagino dando clase, conociendo la manera de aprender de cada uno de los niños y niñas».

La preocupación por la adquisición de conocimientos o la transmisión de información no está vinculada en ningún caso con la sociedad del conocimiento (sobre la que versa la materia de tecnología educativa), en la que estos escolares deberán insertarse. 


\subsection{Perspectiva sobre la enseñanza}

Los estudiantes del grupo 102 tampoco se detienen en cuestiones didácticas. Se observan ejemplos aislados en los que se hace una simple anotación sobre la tarea de enseñar o se postula la intención de proporcionar medios y de usar buenos métodos. Se ven a sí mismos promoviendo una enseñanza atractiva y creativa. Solo en una ocasión se hace referencia a un aula extendida, de puertas abiertas, donde se aprende dentro y fuera del recinto escolar.

Sin embargo, en el grupo 112 ocho estudiantes se refieren de forma explícita a la enseñanza. Los dos varones que lo hacen no apuntan a la enseñanza de contenidos curriculares, sino a la formación de ciudadanos: desarrollar un pensamiento crítico y obtener lo mejor de los escolares que se tengan a cargo. Las seis mujeres que mencionan la enseñanza no la especifican, salvo en uno de los casos en los que se habla de «la materia». En cambio, centran su atención en la forma de enseñar: "con muchas ganas», "con ilusión», "hacer disfrutar», "como me habría gustado que me enseñasen a mí». A título de excepción, se incluyen consideraciones didácticas cuando una alumna se cuestiona "cómo aplicaría distintas metodologías para hacer llegar los conocimientos a los alumnos». Algunas veces, estas manifestaciones biográficas tienen que ver con que fueron estudiantes con bajas calificaciones, incluso participaron en programas de apoyo al final de la escolaridad obligatoria, y ahora quieren ser maestros, según cuentan en los blogs de la materia.

El concepto de formación adquiere mayor relevancia en el grupo 112 que en el 102, trascendiendo al de enseñanza. Las jóvenes estudiantes se piensan ayudando a sus alumnos "a ser mejores» o en la transmisión de "valores», promoviendo a su vez el desarrollo de un "pensamiento crítico», más afín al objetivo de construir ciudadanía en la escuela. Con ese mismo halo de idealización ya comentado se piensa la transmisión de información y valores, «para que la sociedad pueda evolucionar y dejemos de estar alienados por la sociedad». Los varones logran un mayor despliegue de esta misma idea, haciendo referencia a la importancia de formar personas: «ayudarles a sacar lo mejor de sí mismos». Remiten asimismo a fomentar el «espíritu crítico» y a impulsar la «libertad de decisión». Como cabe esperar, formación e implicación son dos nociones solidarias. Y es nuevamente un estudiante varón el que lo pone de manifiesto: «me implico en ellos y su futuro». Otros dos también se ocupan de poner el acento en el proyecto de vida de sus supuestos educandos; la palabra futuro insiste en el discurso de ellos, adjudicándoles distintos destinos. Su mayor alcance corresponde a las expectativas depositadas en quienes serán sus alumnos, siempre que "consigan un futuro mejor para nuestro planeta». Y aunque una estudiante usa el vocablo futuro, lo hace con un propósito autorreferencial: «el gran futuro que me espera».

En general, es sorprendente que, dado el contexto en el que se realiza este estudio (la materia de Tecnología Educativa) y al final de cursar esta materia, esta no forme parte del imaginario de los estudiantes sobre la profesión de maestro. Las tecnologías se ven como un aditamento. Es coherente, por otra 
parte, con lo que pasa con la secuencia metodológica a la que se hizo alusión más arriba, en la que la preparación motivacional para la actividad en clase es el único hito didáctico reseñado por los estudiantes.

\section{Conclusiones}

El estudio puso al descubierto la forma en que encaran la profesión los futuros maestros y, en consecuencia, genera interrogantes sobre la formación que reciben para poder superar cierta idealización que impide, posiblemente, avanzar en otra dirección. Lo que se plantea en los cuestionarios (su posicionamiento sobre la docencia, los niños, el aprendizaje y la enseñanza) entra en diálogo con la perspectiva de la tecnología educativa en la enseñanza que plantea el profesor, la posición de aprendices en la materia y el recorrido realizado desde el comienzo del semestre en que se concreta el estudio.

Tal y como se puso de manifiesto al caracterizar demográfica y socioeducativamente a los grupos, existen diferencias entre los mismos que posiblemente radican en las diferencias en cuanto a la forma de concebir la naturaleza de la profesión y los espacios de intervención. Los miembros del grupo 102, algo más mayores que los del 112, parecen más «vocacionales» al elegir esta formación, algo más rurales, más diversos en sus trayectorias vitales y más ocupados profesionalmente, puesto que simultanean trabajo y estudio. Esto explica, de alguna forma, su adscripción a motivaciones y percepciones profesionales del maestro ideal propias de otras épocas. Además, posiblemente está pesando en el grupo la procedencia de algunos de ellos, es decir, su formación anterior y su actual ocupación laboral (por ejemplo, la de técnico de educación física), así como el deseo de ser maestro especializado en educación física.

Los estudiantes del grupo 112, sin alejarse sustantivamente de un patrón de idealización, parecen ilusionados con su elección. Algo menos presionados por la realidad sociolaboral y las circunstancias de la vida, parecen percibir más matices en la profesión.

En general, se observa entre los estudiantes un proceso de idealización (Laplanche y Pontalis, 1981) por el que se engrandece y se exalta todo aquello que socialmente parece estar ligado al hecho de ser docente. Los problemas y las incertidumbres se minimizan o no se toman en cuenta. Hay multitud de rasgos de bienestar, entusiasmo y fervor en las caracterizaciones realizadas por los estudiantes. Esto nos hace preocupar por lo que ocurrirá cuando contacten con la realidad de las escuelas. También puede estar explicando el tipo de interacción que mantienen con la formación que reciben. Los alumnos parecen estar imbuidos por unos rasgos carismáticos de la profesión, más que por la idea de construir conocimientos en contacto con la comunidad y ayudados por la tecnología, que tiene una clamorosa ausencia en sus respuestas.

La idealización puede transformarse en menosprecio hacia la carrera elegida o hacia el ámbito en el que se desempeña, por lo que emerge un sentimiento de futilidad y desánimo frente a los obstáculos que la realidad impone. En este sentido puede afirmarse que, si bien una cuota de idealización es necesaria 
para emprender un proceso de formación como el que acá se pone en juego, su exceso podría ocasionar en algunos de estos futuros maestros un efecto búmeran y provocar depresión en ellos.

El énfasis puesto por estos jóvenes universitarios en el deber ser es correlacionable con la idealización detectada. Ello genera, probablemente, un estado de ansiedad que no se manifiesta en forma consciente, aunque, según Garrote y Jiménez-Fernández (2018), el nivel de ansiedad en los estudiantes de primer curso es muy bajo. Solo uno de ellos deja entreverlo calificando de «enorme» la responsabilidad que imagina tener sobre sus hombros. Frente a los estímulos ansiógenos, los mecanismos defensivos tienden entonces a rigidizar el pensamiento y las prácticas docentes pierden esa flexibilidad necesaria para que el acto creativo tenga lugar, lo que ya fuera enunciado como gesto espontáneo o como lo más auténtico del ser (Winnicott, 1992). El impulso creador o el vivir creador es aquí concebido como un estado saludable, como aquello que le permite al individuo sentir que la vida es algo significativo, que vale la pena de ser vivida. Apelar a esta potencialidad humana en ámbitos educativos revierte sobre el docente en un sentimiento de bienestar sobre su quehacer profesional. Se opone a esta vivencia creadora una relación enfermiza con la realidad, un vínculo de acatamiento, donde debería ubicarse el deber ser: el mundo exterior es percibido entonces como aquello a lo que hay que adaptarse. En estas circunstancias no hay cambio posible, solo un sentimiento de inutilidad y resignación. Un fuerte anclaje en el deber ser constituye siempre un riesgo para quienes deberán enfrentarse con las complejas y cambiantes cotidianeidades del aula escolar.

La cuestión sobre la que pensar es qué hacer con las expectativas de estos maestros en formación, cómo hacer para que la brecha entre sus ideales y la realidad escolar a la que habrán de enfrentarse no sea desmedida y provoque en el futuro fenómenos adversos a un buen desempeño docente. Unido a ello, es preciso tomar en cuenta que los ideales no se enseñan ni se prescriben, en sentido estricto, sino que son parte de la subjetividad de las personas, de los colectivos humanos y hasta de las diferentes épocas. $\mathrm{Al}$ mismo tiempo, los currículos de formación docente no contemplan la implementación de espacios formales o informales, obligatorios u opcionales, de reflexión sobre estas representaciones preconscientes sobre las que se construye una figura profesional imaginaria e idealizada a la que será necesario renunciar, lo que es posiblemente un desafío pendiente de consideración.

Todo ello debería hacer replantear la docencia vinculada a la formación de formadores. Se impone observar las prácticas en las que ocurre la enseñanza, negociar la participación en proyectos en los que se analicen sus resultados y fomentar la apertura a la creación, lo que supone aceptar nuevas incertidumbres y ser docente de una forma que ahora no se sospecha. La tecnología educativa podría ser una de las materias que les ayudara a generar esas participaciones negociadas en la realidad, pero ello tiene que ver más con el ajuste de los medios a las condiciones del contexto (en forma de proyectos, de partida abiertos a resultados imprevistos) que con la prescripción. 


\section{Referencias bibliográficas}

Benejam, P. (1986). La formación de maestros: Una propuesta alternativa. Barcelona: Laia.

Esteban, F. y Mellen, T. (2016). ¿Por qué quieres ser maestro?, ¿Cómo es un buen maestro?: Ideas para la formación universitaria. Bordón: Revista de Pedagogía, 68(2), 185-198.

Esteve, J.M. (1998). La aventura de ser profesor. Cuadernos de Pedagogía, 266, 46-50.

Gallardo, N. y Vázquez, A. (2016). ¿Por qué ser maestro? Motivaciones y expectativas del estudiante de Magisterio. Revista de estudios extremeños, 72(3), 1661-1696.

Garrote, D. y Jiménez-Fernández, S. (2018). Perfil motivacional en estudiantes del grado de Maestro en Educación Infantil y Primaria. Educar, 54(2), 351-368. <https://doi.org/10.5565/rev/educar.899>

Goodson, I.; Biesta, G.; Tedder, M. y Adair, N. (2010). Narrative learning. Nueva York: Routledge.

Hernández, F. (2008). Deconstruir la figura del asesor: Una revisión desde los márgenes. Profesorado: Revista de Currículum y Formación del Profesorado, 12(1), 1-14.

Joyce, B. y Clift, E. (1984). The Phoenix Agenda: Essential Reform in Teacher Education. Educational Researcher, 13(4), 5-18.

Laplanche, J. y Pontalis, J.B. (1981). Diccionario del psicoanálisis. Barcelona: Labor.

LATORre, A. (2003). La investigación-acción: Conocer y cambiar la práctica educativa. Barcelona: Graó.

Lundgren, U. (1988). Nuevos desafíos para los profesores y para una formación del profesorado. Revista de Educación, 285, 291-328.

Medina, J.L. y Pérez Cabrera, M.J. (2017). La construcción del conocimiento en el proceso de aprender a ser profesor: La visión de los protagonistas. Profesorado: Revista de Curriculum y Formación del Profesorado, 21(1), 17-38.

Paredes-Labra, J.; Esteban, R.M. y Fernández Prieto, M.S. (2016). El Prácticum de Maestro en las voces de sus tutores: Balance del plan 2010 en la UAM. Revista Complutense de Educación, 27(1), 161-178.

Sánchez Lisson, E. (2009). Mitos y realidades en la carrera docente. Revista de Educación, 348, 465-488.

Sancho, J.; Hernández, F; Larraín, V. y Montané, A. (2011). La entrevista como espacio de relaciones en una investigación planteada como acompañamiento. En J.I. Rivas, F. Hernández, J.M. Sancho y C. Núñez (eds.). Historias de vida en educación: Sujeto, diálogo, experiencia (pp. 153-160). Barcelona: Universidad de Barcelona.

SAN Román, S. (2019). Las primeras maestras: Orígenes del proceso de feminización docente en España. Barcelona: Ariel.

Santos Guerra, M.A. (1993). La formación inicial. Cuadernos de Pedagogía, 220, $50-54$.

Schön, D. (1983). The Reflective Practitioner: How professionals think in action. Londres: Temple Smith.

TARDIF, M. (2004). Los saberes del docente y su desarrollo profesional. Madrid: Narcea. WinnicotT, D.W. (1992). Realidad y juego. Barcelona: Gedisa. 